\title{
The effect of synovium graft on conjunctiva in rabbit model of dry eye
}

\author{
Keungjin Park ${ }^{1, \#}$, Kyungmin Nam ${ }^{1, \#}$, Min Jang ${ }^{1}$, Won-Jae Lee ${ }^{1}$, Seong Mok Jeong ${ }^{2}$, Seulgi Bae ${ }^{1}$, \\ Young-Sam Kwon ${ }^{1}$, Sae-Kwang $\mathrm{Ku}^{3}$ and Sungho Yun ${ }^{1, *}$ \\ ${ }^{1}$ College of Veterinary Medicine, Kyungpook National University, Daegu 41566, Korea \\ ${ }^{2}$ College of Veterinary Medicine, Chungnam National University, Daejeon 34134, Korea \\ ${ }^{3}$ Department of Anatomy and Histology, College of Korean Medicine, Daegu Haany University, Gyeongsan 38610, Korea
}

Received January 26, 2021

Revised March 24, 2021

Accepted March 24, 2021

\section{*Correspondence}

Sungho Yun

E-mail: shyun@knu.ac.kr

\section{ORCID}

https://orcid.org/0000-0002-9027-3859

"These authors contributed equally to this work.

\begin{abstract}
This study was performed to identify the effect of synovium graft on conjunctiva in rabbits after dry eye induction. Six New Zealand White rabbits were used as dry eye models. Both eyes were divided to two groups as control and synovium graft group. The synovium graft was performed in fourth week after dry eye modeling. Quantitative change of tears through Schirmer tear test (STT), qualitative change of tear film through tear film break up time (TFBUT), and damage of cornea through fluorescein staining were observed for 10 weeks at intervals of two weeks. Histological examination was performed to evaluate cornea and conjunctiva at tenth week. In both groups, STT and TFBUT were significantly decreased in 4 weeks after modeling compared to 0 weeks $(p<0.05)$. After synovium graft, there were increases in STT value at 4 weeks and TFBUT at 4 and 6 weeks in graft group $(p<0.05)$. Corneal fluorescein staining showed no significant difference between the two groups. In histopathological examination, grafted synovium was detected as round to ovoid ingression folds, well attached to grafted regions with $0.11 \pm 0.04 \mathrm{~mm}^{2}$ (range, 0.05 $0.16 \mathrm{~mm}^{2}$ ). These results indicated that the synovium graft on the conjunctiva had an effect on the qualitative and quantitative improvement of the tear film even though there was no histological change.
\end{abstract}

Keywords: dry eye, keratoconjunctivitis sicca, rabbit, synovium graft

\section{INTRODUCTION}

Dry eye, also known as keratoconjunctivitis sicca, is a common ocular surface disease in dogs showing ocular discomfort with hyperemia, a mucoid to mucopurulent exudate, pigmentation, corneal vascularization, and sometimes corneal ulcer (Sanchez et al., 2007). It is caused by one or more of tear film layer problem. The major causes of breaking this layer are the decrease of tear secretion and the increase of tear evaporation. As a result, the dehydration of tear film as well as hyperosmolarity of tear occurs. Hyperosmolarity activates inflammatory response and releasing of inflammatory mediators into the tears. This causes damage to the ocular surface epithelium (The definition and classification, 2007).

The rabbit dry eye model has been well established and evaluated from 1987 (Gilbard et al., 1987). Because of the large exposed ocular surface in rabbits compared with mice, standard dry eye clinical tests such as tear film break-up time (TFBUT), Schirmer tear test (STT) and fluo- 
rescein staining of the ocular surface can be much more easily performed in rabbits (Barabino and Dana, 2004). In a recent study, a mild dry eye model is established by surgically removing the lacrimal gland, harderian gland, and nictitating membrane, and a moderate dry eye model is established by chemical burning bulbar conjunctiva. By combining above both techniques, a severe dry eye model is established (Li et al., 2013).

Medications are the primary therapy of dry eye. But there are some disadvantages of the medical treatments, including frequent application and long administration periods. In some cases of dry eye, surgical intervention are indicated, including parotid duct transposition and partial tarsorrhaphy (Plummer et al., 2008; Rhodes et al., 2012). However, these surgical methods are limited in their application and have complications like dysfunction of parotid duct or mineral precipitates on ocular surface (Plummer et al., 2008; Rhodes et al., 2012). Therefore, other surgical methods such as transplants of labial mucosa or nasal mucosa have been tried for the treatment of the dry eye (Castanho et al., 2013; Wenkel et al., 2000).

It was reported that synovial fluid, tear, and saliva have similar functions (Jay and Hong, 1992). Synovial fluid contains lubricants such as hyaluronic acid synthesized by the synovial membrane and lubricin secreted by synovial fibroblast (Jay et al., 2000). Numerous studies demonstrated that hyaluronic acid accelerates corneal wound healing both in vitro and in vivo (Yang et al., 2010; Wu et al., 2013). Recent studies showed that lubricin is secreted by meibomian gland in rabbits and ocular surface epithelia in human (Cheriyan et al., 2011; Schmidt et al., 2013). In human, lubricin had effective improvement in clinical symptoms of dry eye compared with hyaluronic acid (Lambiase et al., 2017). In synovial fluid and synovium, it is also detected bone morphogenetic protein that is important for the development and restore of lacrimal glands and has functions for corneal tissue repair (Saika et al., 2005; Zoukhri et al., 2008).

There has not been reported the effect of synovium graft on conjunctiva in dry eye. The purpose of this study is to evaluate the effect of synovium graft on conjunctiva to treat experimentally induced dry eye in rabbits.

\section{MATERIALS AND METHODS}

\section{Animals}

Six healthy male New Zealand White rabbits (age: 12 weeks) without any ophthalmic diseases were used. All experimental protocols were approved by the Institutional Animal Care and Use Committees of Kyungpook National University (2019-0161).

\section{Study design}

Dry eye was induced by surgical excision of lacrimal gland, Harderian gland and nictitating membrane. The clinical examinations during dry eye modeling were performed every other week for 4 weeks. After that, in all of the 6 rabbits, the right eyes and the left eyes were used for Group A (control) and Group B (treatment), respectively. Both eyes were dry eye-induced and then the left eye received a synovium graft surgery on conjunctiva. In both group, clinical examinations were performed every other week for 6 weeks and histological examination was performed after being euthanized at 6 week.

\section{Dry eye modeling}

Each rabbit was anesthetized and positioned in lateral recumbency. For aseptic surgery, all surgical procedures were performed in positive pressure operating room. Then the periocular surface and left stifle were prepared by shaving and sterilizing with alcohol and chlorhexidine. The globe was flushed with $0.2 \%$ povidone iodine solution. Before surgery, local anesthesia was performed with Alcaine ${ }^{\circledR}$ eye drop $(0.5 \%$ proparacaine hydrochloride ophthalmic solution, Alcon Laboratories, Belgium).

Temporal lobes of the lacrimal gland were removed by blunt dissection of lateral canthal region. After blunt dissection of intraorbital tissues, infraorbital lobes of the lacrimal gland were gently drawn with adson-brown forceps. The Harderian glands were separated by blunt and sharp dissection. Then, both of nictitating membranes and Harderian glands were removed in their entirety, together with the complete capsule (Fig. 1).

\section{Synovium graft on conjunctiva}

After anesthesia, surgical site was aseptically prepared and the synovium graft was taken from the stifle joint by craniolateral approach. Using $8 \mathrm{~mm}$ biopsy punch (Miltex, Inc., York, PA, USA), a graft was obtained from the inner 

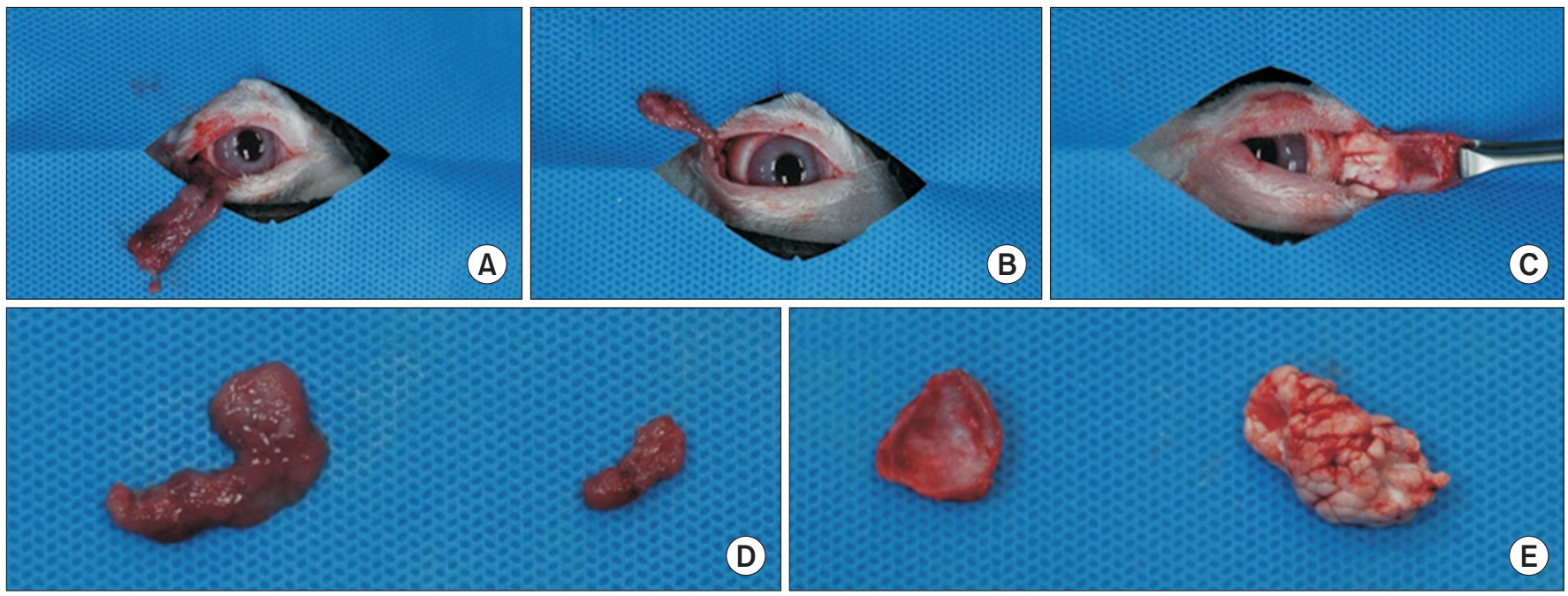

Fig. 1. Surgical steps of inducing dry eye. (A) Resecting infraorbital lobe of lacrimal gland, (B) Resecting temporal lobe of lacrimal gland, (C) Removing nictitating membrane and Harderian gland at the medial canthus region, (D) Surgically resected infraorbital lobe (left) and temporal lobe (right) of lacrimal glands, (E) Resected nictitating membrane (left) and Harderian gland (right).
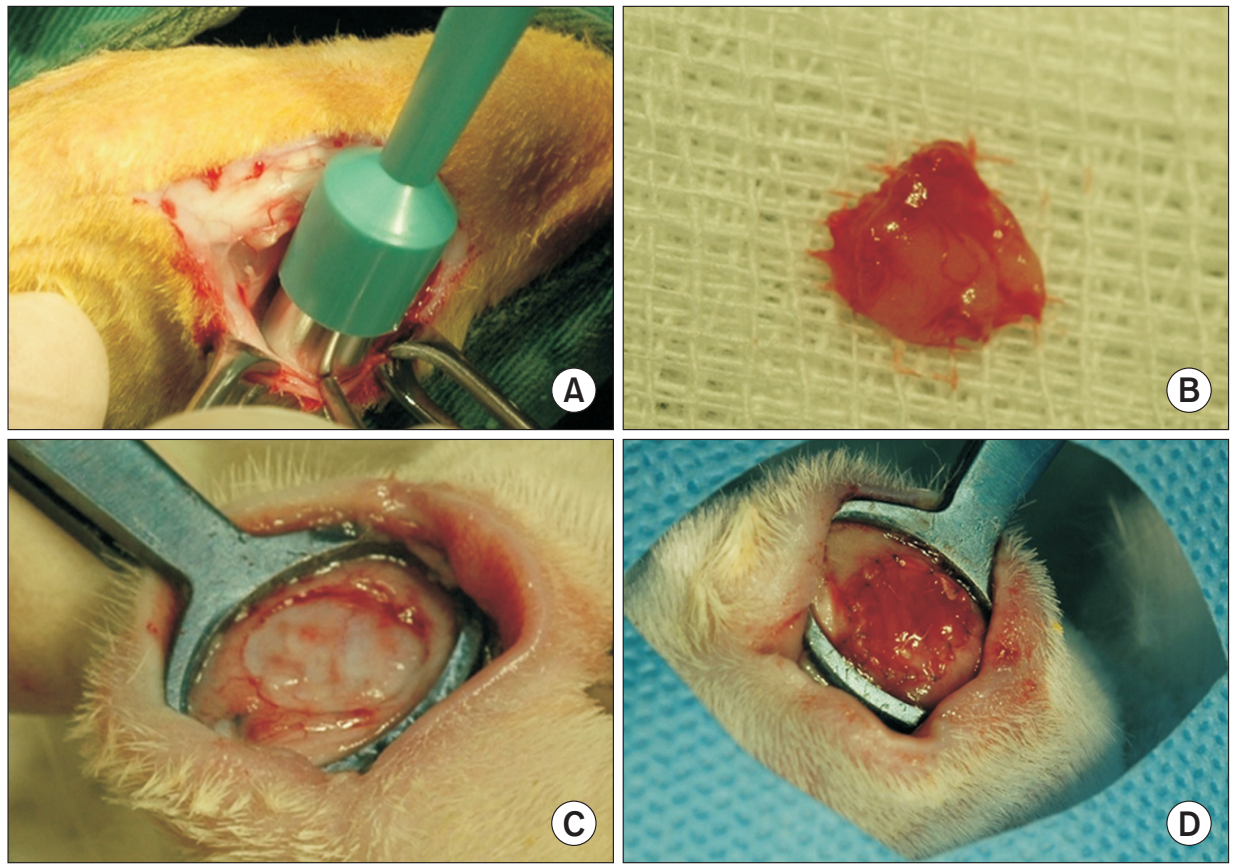

Fig. 2. Synovium graft on conjunctiva. (A) Excision of synovium from stifle joint, (B) Excised synovium graft, (C) Preparing the recipient site in the palpebral conjunctiva, (D) Suture of synovium graft on conjunctiva.

part of joint capsule, and the incision is closed in layers. The globe and conjunctiva were flushed with $0.2 \%$ povidone iodine solution. Before surgery, Alcaine ${ }^{\circledR}$ eye drop was administrated for local anesthesia. The palpebral conjunctiva was everted using a chalazion clamps. Then using same biopsy punch and metzenbaum scissors, palpebral conjunctiva was detached and the synovium graft was sutured on that site using simple interrupted suture methods with absorbable suture size 8-0 (KRUUSE Sacryl suture, KRUUSE ${ }^{\circledR}$, Denmark) (Fig. 2). Intramuscular tramadol (2 mg/kg, Maritrol ${ }^{\circledR}$; Je Il Pharm. Co. Ltd., Korea) and cefazolin $\left(25 \mathrm{mg} / \mathrm{kg}\right.$, Cefazol ${ }^{\circledR}$, Hangook Chorus Pharm, Korea) were given twice a day for 5 days after surgery.

\section{Clinical examination}

STT was used for checking the amount of tear. Without any eye drops, one Schirmer strips (Schirmer Mark Blu, Optitech Eye care ${ }^{\circledR}$, India) was inserted into the middle 
of lower eyelid and eyes were closed. After one min, the measured values were recorded. TFBUT was performed for examination the tear film stability. After applying fluorescein to the ocular surface by a wetted fluorescein strip, the time from opening the eyes to the appearance of the first spot in the corneal surface was measured in seconds. Interval over $4 \mathrm{hr}$ was given between STT and TFBUT. Fluorescein staining was checked after TFBUT examination. Using slit-lamp microscope with a cobalt blue filter, the cornea of all rabbits was taken a photograph at a magnification of 10. The National Eye Institute/Industry grading scale (NEI) was used for scoring of corneal staining. The cornea was divided into five zone (center, superior, temporal, nasal and inferior zones), and scored from 0 to 3 (Grade 0: normal; Grade 1: mild, superficial stippling; Grade 2: moderate, punctate staining including superficial abrasion of the cornea; Grade 3: severe, corneal erosion or deep corneal abrasion). The maximum possible clinical score was 15 (Ward, 2008).

\section{Histological process}

Under the sedation with intramuscular injection of xylazine (3 mg $/ \mathrm{kg}$, Rompun ${ }^{\circledR}$; Bayer Korea, Korea), euthanasia was performed with intravenous injection of T-61 euthanasia solution (American Hoechst, USA). To eliminate the pain of the experimental animal, the whole globe was enucleated from all rabbits after euthanasia, using the lateral subconjunctival enucleation technique. Palpebral conjunctiva of the upper eyelid, where synovium was grafted, was excised widely. For histological examination, all samples were fixed in $10 \%$ neutral formaldehyde

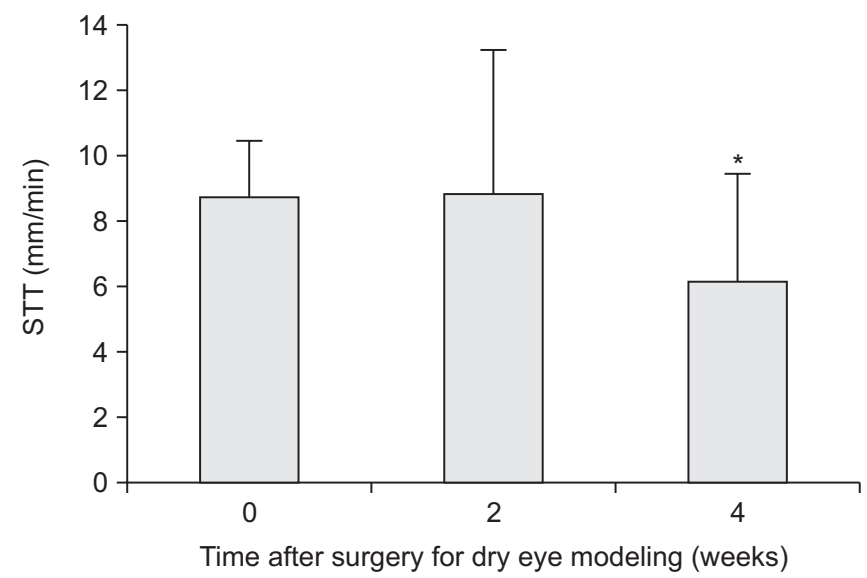

Fig. 3. The Schirmer tear test (STT) value during inducing dry eye. Data were expressed as mean \pm SD. *Significant differences compared with 0 week $(p<0.05)$. solution. Each upper palpebral conjunctiva was crossly trimmed as one part based on the center located graft sites, and the sample of each cornea was separated from individual globes and crossly trimmed also as one part. After that, they were embedded in paraffin and sectioned $(3-4 \mu \mathrm{m})$ as one section in cornea and as two serial sections in upper eyelid, respectively. Representative sections were stained with hematoxylin and eosin staining (HE) for general histology, and periodic acid Schiff (PAS) for detecting mucosubstances. The histological profiles were interpreted under a light microscope (Model Eclipse $80 \mathrm{i}$, Nikon ${ }^{\circledR}$, Japan) as blinds to group distribution when this analysis was made.

\section{Histomorphometrical analysis}

To observe more detail histopathological changes, remnant grafted synovium area $\left(\mathrm{mm}^{2}\right)$ and mean epithelial thickness $(\mu \mathrm{m})$ and mean numbers of PAS positive cells (numbers $/ \mathrm{mm}^{2}$ ) around graft and peri-graft regions of upper palpebral conjunctiva were calculated using a computer-based automated image analyzer (iSolution FL ver 9.1, IMT i-solution Inc., Canada) according to the previous report with some modifications (Kim et al., 2015a). In addition, mean thicknesses $(\mu \mathrm{m})$ of total cornea, epithelial and stroma were also calculated by an automated image analyzer, according to previous reports with some modifications (Kim et al., 2015b).

\section{Statistical analysis}

All data were recorded as mean \pm standard deviation (SD). To compare between groups and times, non-para-

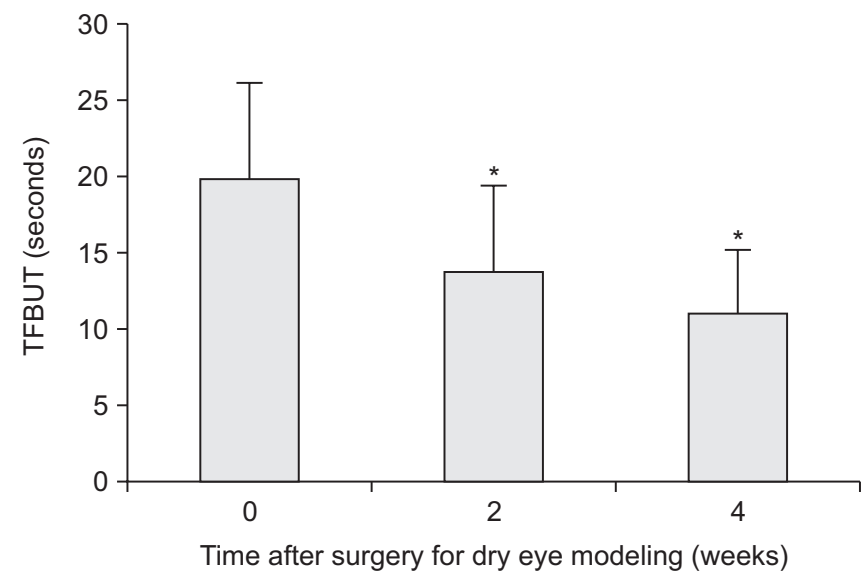

Fig. 4. The tear film break-up time (TFBUT) during inducing dry eye. Data were expressed as mean \pm SD. *Significant differences compared with 0 week $(p<0.05)$. 
metric Mann-Whitney U test was performed using SigmaPlot (Version 14.0, Systat Software Inc, UK). $p$ values less than 0.05 were considered statistically significant.

\section{RESULTS}

\section{Ophthalmic examinations during inducing dry eye}

Preoperative STT value and TFBUT made no difference between both eyes. At 4 weeks after induction of dry eye, the STT value was significantly lower compared with preoperative value $(p<0.05)$ (Fig. 3). In addition, TFBUT was significantly shorten in 2 and 4 weeks than 0 week ( $p$ $<0.05$ ) (Fig. 4). Post-operative corneal fluorescein staining score at 2 and 4 weeks was significantly higher than 0

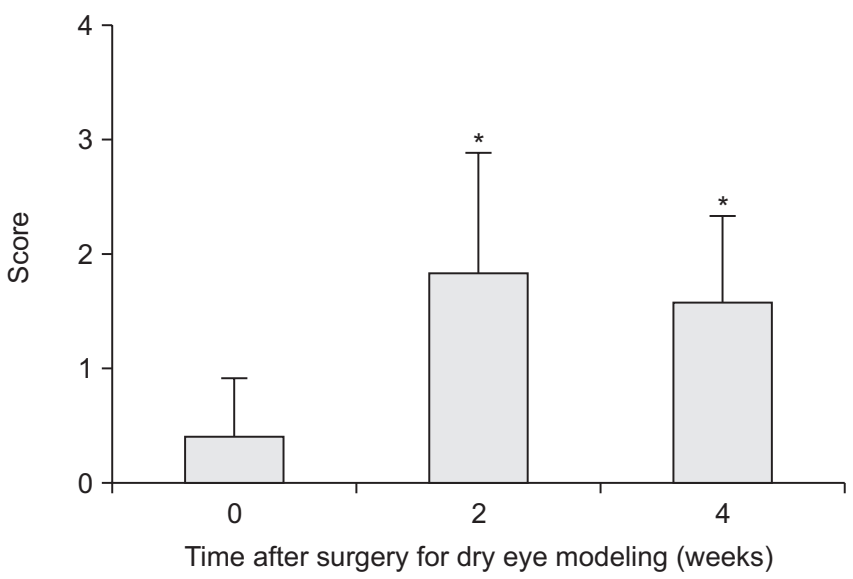

Fig. 5. The corneal fluorescein staining score during inducing dry eye. Data were expressed as mean \pm SD. ${ }^{*}$ Significant differences compared with 0 week $(p<0.05)$.

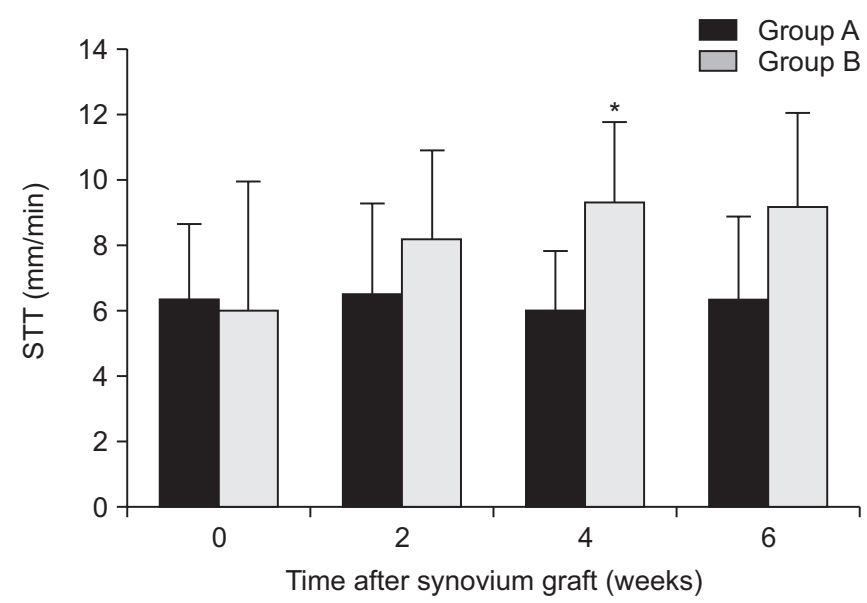

Fig. 6. The Schirmer tear test (STT) value after synovium graft. Data were expressed as mean \pm SD. Group A: control group, Group B: treatment group. *Significant differences compared with Group A in same weeks $(p<0.05)$. week $(p<0.05)$ (Fig. 5).

\section{Ophthalmic examinations after treatment}

All ophthalmic examinations were performed at baseline then every other week. Preoperatively, no significant difference in ophthalmic examinations were identified between the groups. In group B, the STT value was gradually increased during 0 to 4 weeks, but had no significant differences among the weeks. In 4 weeks, the STT value of group B was significantly higher than that of the group A $(p<0.05)$ (Fig. 6). The TFBUT of group B were significantly higher than that of the group A at 4 and 6 weeks ( $p$ $<0.05$ ) (Fig. 7). In comparison between both groups, there was no significant difference of the corneal fluorescein

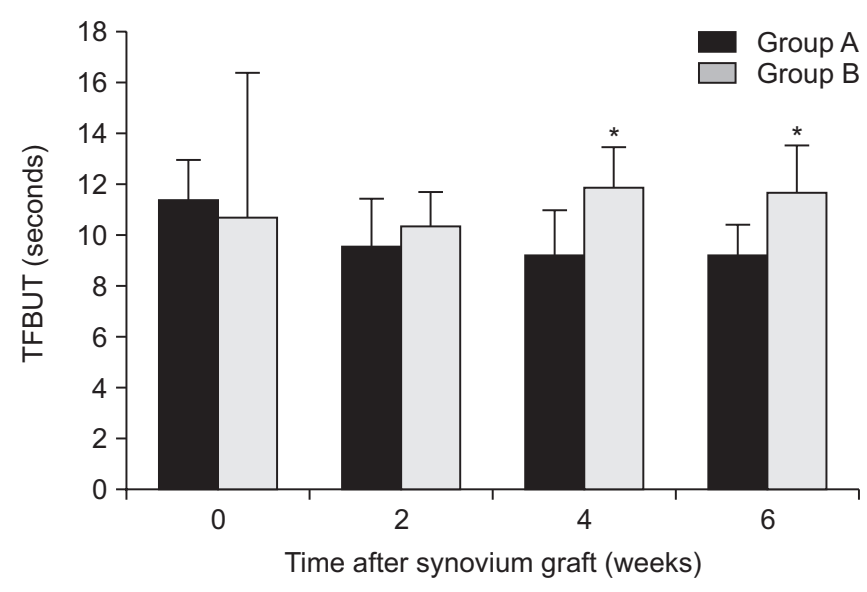

Fig. 7. The tear film break-up time (TFBUT) after synovium graft. Data were expressed as mean \pm SD. Group A: control group, Group B: treatment group. *Significant differences compared with Group A in same weeks $(p<0.05)$

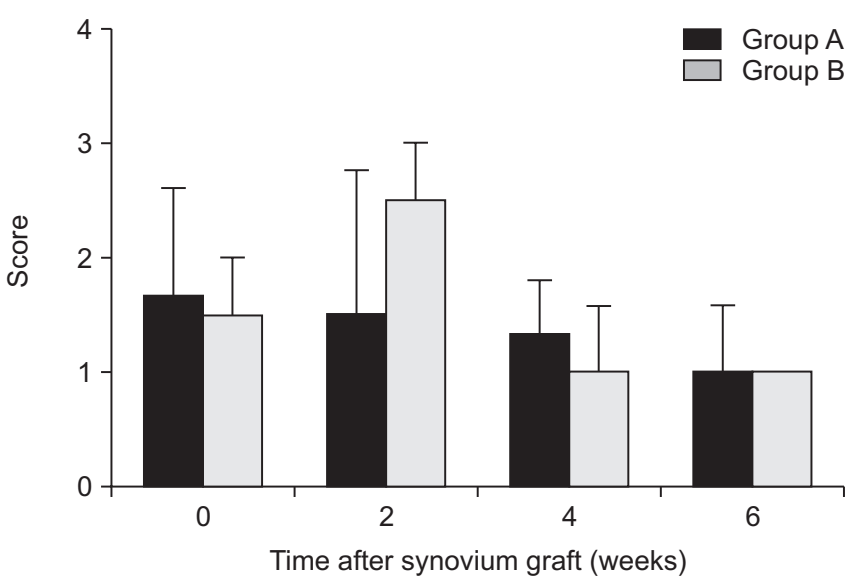

Fig. 8. The corneal fluorescein staining score after synovium graft. Data were expressed as mean \pm SD. Group A: control group, Group B: treatment group. 
staining score. Although, the corneal staining scores in both group A and B were gradually decreased, there was an increase in group B at 2 weeks but without any significant differences (Fig. 8).

\section{Histopathological analysis}

At histopathological observation, grafted synovium was detected as round to ovoid ingression folds, well attached to grafted regions, with $0.11 \pm 0.04 \mathrm{~mm}^{2}$ (range, 0.05-0.16 $\mathrm{mm}^{2}$ ) of mean remnant grafted synovium area. However, no significant changes on the mean epithelial thicknesses and PAS positive cell numbers were demonstrated in group B as compared to group A, respectively (Table 1, Fig. 9). In addition, there were no significant changes in the mean thicknesses of total cornea, epithelium and stroma in group B when compared to group A (Table 2, Fig. 10).

\section{DISCUSSION}

The rabbit dry eye model used in this study was induced by surgical removal of all of the nictitating membrane, lacrimal glands, and Harderian glands, which resulted in the decrease of aqueous and lipid layers of tear film. In

Table 1. The histomorphometrical analysis on the conjunctiva

\begin{tabular}{lcrr}
\hline \multicolumn{1}{c}{ Index (unit) } & Group A & \multicolumn{2}{c}{ Group B: regions } \\
\cline { 3 - 4 } & & Graft & Peri-graft \\
\hline Remnant synovium graft areas $\left(\mathrm{mm}^{2}\right)$ & $0.00 \pm 0.00^{+}$ & $0.11 \pm 0.04$ & $0.00 \pm 0.00^{\dagger}$ \\
Mean epithelial thicknesses $(\mu \mathrm{m})$ & $22.72 \pm 2.37$ & $21.95 \pm 1.95$ & $22.69 \pm 2.21$ \\
Mean PAS positive cell numbers $\left(\right.$ cells $\left./ \mathrm{mm}^{2}\right)$ & $77.33 \pm 15.16$ & $66.67 \pm 17.00$ & $77.00 \pm 10.49$ \\
\hline
\end{tabular}

Group A: control group, Group B: treatment group. Values are expressed as mean \pm SD of 6 histological fields. ${ }^{\dagger}$ Not detected.

HE

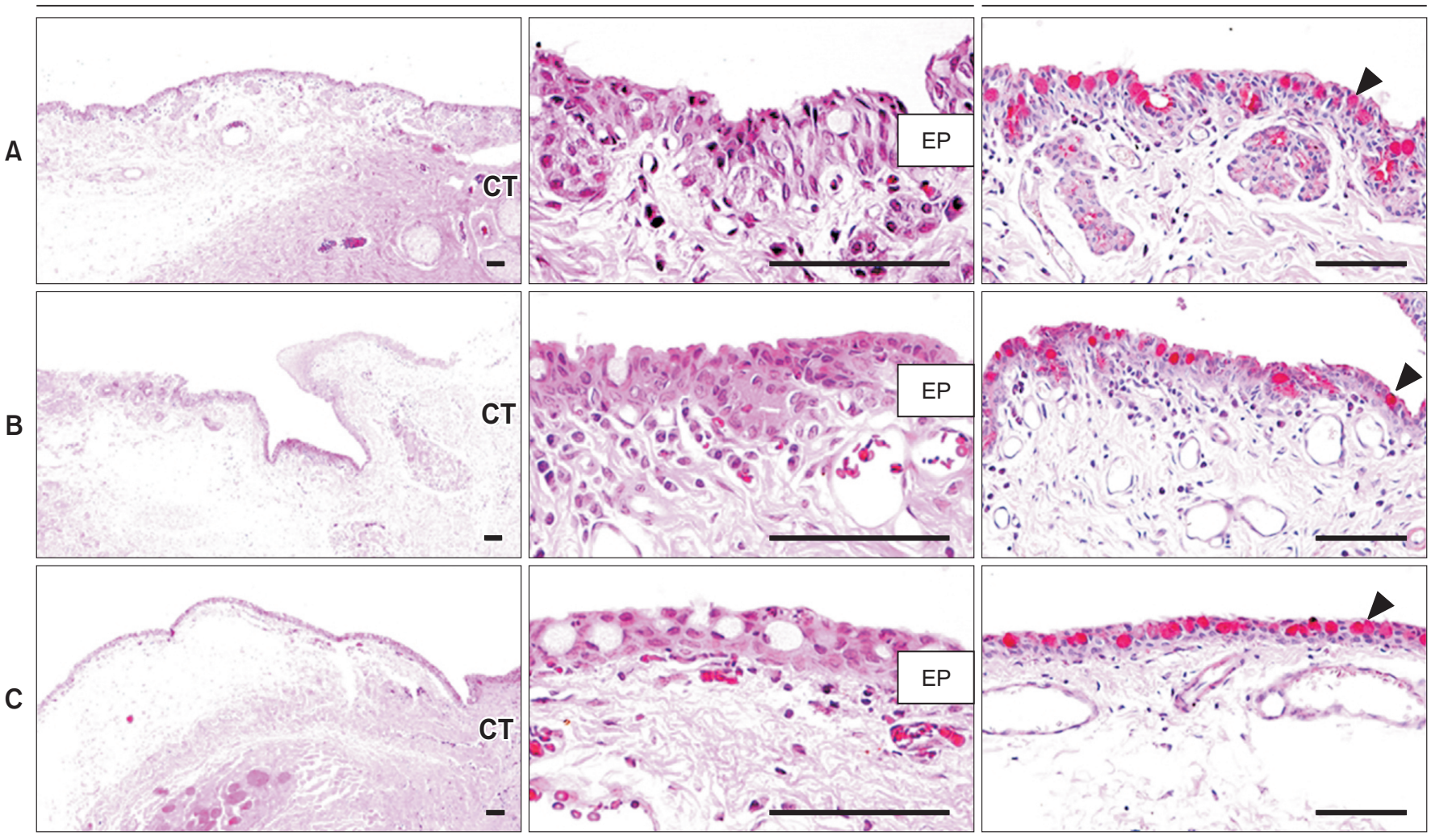

Fig. 9. The representative histopathological profiles of the upper eyelid conjunctiva tissues. Histopathological examination was conducted on sixth week after graft. (A) Group A: control group, (B) Group B: Graft site of treatment group, (C) Group B: Peri-graft site of treatment group. All HE stains, scale bars $=80 \mu \mathrm{m}$. CT: Conjunctiva, EP: Epithelium, Black arrowhead: PAS positive. 
previous studies, significant decrease in tears, elevation of tear osmolarity, mild damage in cornea, and relatively small decrease of goblet cell density were shown within four weeks in this model (Gilbard et al., 1987; Li et al., 2013). In this study, significantly decreased STT and TFBUT values, and increased corneal staining scores were noted at four weeks for dry eye modeling, which was regarded that the mild dry eye was induced.

It was hypothesized that the components of the synovial fluid from the grafted synovium would replenish the insufficiency of aqueous layer and reinforce mucin layer in this rabbit model. As a result of this, it was expected that the quantity and quality of tear would be improved. In this study, the STT values of control group were remained constantly lower than pre-operative values, but a gradual increases of the STT value were observed as time passed in treatment group. When compared with the control group, it was found that the STT value in the treatment group increased significantly in the four weeks after graft-

Table 2. The histomorphometrical analysis on the cornea

\begin{tabular}{lcc}
\hline \multicolumn{1}{c}{ Index (unit) } & Group A & Group B \\
\hline Thicknesses $(\mu \mathrm{m})$ & & \\
Total & $353.50 \pm 22.86$ & $355.50 \pm 23.46$ \\
Epithelial & $29.24 \pm 2.29$ & $28.33 \pm 2.06$ \\
Stroma & $314.12 \pm 25.81$ & $316.35 \pm 24.80$ \\
\hline
\end{tabular}

Group A: control group, Group B: treatment group. Values are expressed as mean \pm SD of 6 histological fields. ing. Increasing of STT value means that it is due to the increase of tear production or improvement of the storing ability (Yokoi et al., 2004). TFBUT is to measure the time when a tear film begins to break after it is distributed on the surface of cornea. The decrease in this value is caused by the instability of tear film. In this study, the TFBUT in treatment group increased significantly compared to control group in 4 weeks and 6 weeks of graft. Increasing of this value means that the instability of the tear film has been alleviated by supplement of mucin layer (Davidson and Kuonen, 2004). The increases in both STT and TFBUT may be due to the effect of components secreted from grafted synovium in this study. The increase of tear quantity may be due to reflex tears, which were produced by exposure of the eye to external factors, as irritation of the graft suture material in this study (Gelatt et al., 2013). However, a correlation between eye irritation and TBUT reduction has been reported, and in addition, an increase in STT values and a decrease in TFBUT were observed in eyes exposed to chronic irritation (Satici et al., 2003).

The accurate assessment of the status of ocular surface is important to clinician, and fluorescein staining is useful for detecting various corneal abnormalities such as corneal wound, fistula and ulcer (Ward, 2008). In this study, corneal fluorescein staining scores showed statistically significant increases after dry eye modeling. After synovium graft, the highest score shown at second week of treatment group was less than 3 and have no signifi-

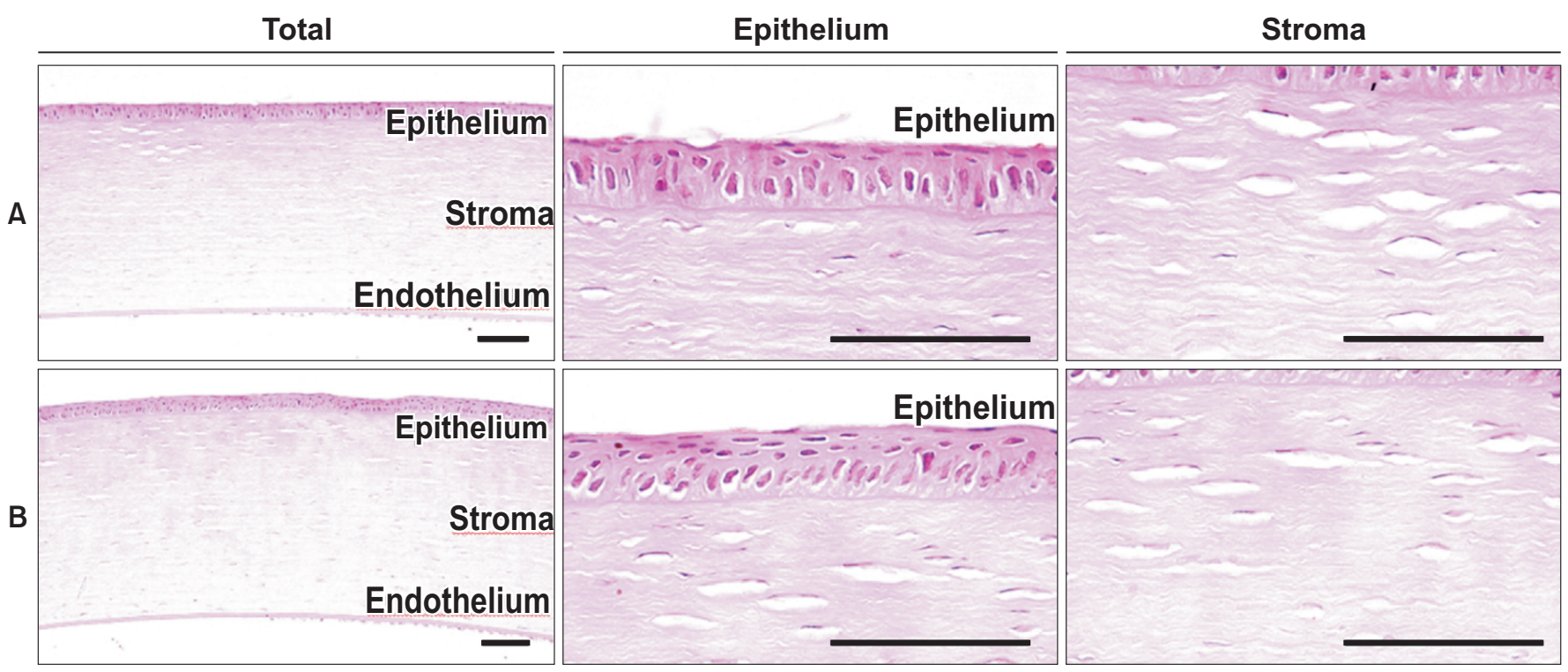

Fig. 10. The representative histopathological profiles of the cornea tissues. Histopathological examination was conducted on sixth week after graft. (A) Group A: control group, (B) Group B: treatment group. All HE stains, scale bars $=80 \mu \mathrm{m}$. 
cant difference between groups. Considering that the total clinical score is 15 , corneal damage was mild. The location of the corneal staining was limited to counterpart region of synovium graft, and the staining was observed until second week after graft. Accordingly, it is thought to be a minor postoperative complication. To reduce this complication, like other studies, it could be considered that changing the location of the graft into a conjunctival fornix or using of therapeutic contact lens (Kuckelkorn et al., 1996; Sant' Anna et al., 2012).

In this study, grafted synovium region was detected with $0.11 \pm 0.04 \mathrm{~mm}^{2}$ (range, 0.05-0.16 $\mathrm{mm}^{2}$ ). Because the remnant synovium was shrunk, it is necessary to precede advanced graft techniques to improve the survival rate of graft tissue through preventing graft shrinkage by applying a larger tissue or scaffold when performing synovium graft on the conjunctiva. The synoviocytes build multiple layers and show morphological changes to a cuboidal shape, indicating that proliferation and hypertrophy have occurred (Laverty et al., 2010). Although it was reported that the synovial fluid with arthritis contains more lipids or growth factors, the precise effect of these morphological changes on function is unknown (Bole, 1962; Lettesjö et al., 1998). Therefore, further studies are needed to evaluate the changes in tear components in dry eye-induced and synovium grafted eyes.

Histopathological features of dry eye commonly includes epithelium damages of cornea and conjunctiva, desquamation of stroma and surface epithelial linings in the cornea, and decrease of goblet cells in the conjunctival epithelium (Nakamura et al., 2003; Higuchi et al., 2007). In this study, there was no difference in histological examination of conjunctiva and cornea between groups. It was suggested that there was insufficient therapeutic effect of synovium graft or that the ocular surface was not severely damaged in this model to see the therapeutic effect. Because all eyes of rabbits in this study were induced to dry eye, there was no comparable histological data of normal rabbit in this study, however, there was no remarkable difference compared to normal corneal thicknesses reported in other studies $(373 \pm 7.2 \mu \mathrm{m}$ to $407 \pm$ $20 \mu \mathrm{m}$ ) (Chan et al., 1983; Wang et al., 2014). Therefore, long-term follow-up of synovium grafts or investigations in more severe dry eye models should be performed as further studies.

\section{CONCLUSION}

In conclusion, the present study suggests that the quantity and quality of tear are significantly improved by synovium graft on the conjunctiva. However, more advanced surgical techniques and post-operative medications to increase survival rate of grafted synovium seemed to be required. The evaluations of tear components are also needed for understanding the precise effect of synovium graft.

\section{CONFLICTS OF INTEREST}

No potential conflict of interest relevant to this article was reported.

\section{AUTHOR CONTRIBUTIONS}

Conceptualization: Young-Sam Kwon, Sungho Yun

Animal works: Keungjin Park, Kyungmin Nam

Investigation: Seulgi Bae, Min Jang

Methodology: Won-Jae Lee

Formal analysis: Sae-Kwang Ku

Software: Won-Jae Lee

Supervision: Seong Mok Jeong

Writing original draft: Keungjin Park, Kyungmin Nam

Review \& editing: Young-Sam Kwon, Sungho Yun

\section{AUTHOR'S POSITION AND ORCID NO.}

\author{
K Park, MS, \\ https://orcid.org/0000-0001-6433-2141 \\ K Nam, MS, \\ https://orcid.org/0000-0001-6139-5438 \\ M Jang, Assistant Professor, \\ https://orcid.org/0000-0002-2188-1906 \\ WJ Lee, Assistant Professor, \\ https://orcid.org/0000-0003-1462-7798 \\ SM Jeong, Professor, \\ https://orcid.org/0000-0001-8653-5927 \\ $S$ Bae, Assistant Professor, \\ https://orcid.org/0000-0001-9487-5665 \\ YS Kwon, Professor, \\ https://orcid.org/0000-0002-6489-0327 \\ SK Ku, Professor, \\ https://orcid.org/0000-0003-1269-3804
}


S Yun, Assistant Professor, https://orcid.org/0000-0002-9027-3859

\section{REFERENCES}

Barabino S and Dana MR. 2004. Animal models of dry eye: a critical assessment of opportunities and limitations. Invest. Ophthalmol. Vis. Sci. 45:1641-1646.

Bole GG. 1962. Synovial fluid lipids in normal individuals and patients with rheumatoid arthritis. Arthritis Rheum. 5:589601.

Castanho LS, Moreira H, Ribas CAPM, Wouk AFPF, Sampaio M, Giordano T. 2013. Labial salivary glands transplantation in the treatment of dry eye in dogs by autograft. Rev. Bras. Oftalmol. 72:373-378.

Chan T, Payor S, Holden BA. 1983. Corneal thickness profiles in rabbits using an ultrasonic pachometer. Invest. Ophthalmol. Vis. Sci. 24:1408-1410.

Cheriyan T, Schmid TM, Spector M. 2011. Presence and distribution of the lubricating protein, lubricin, in the meibomian gland in rabbits. Mol. Vis. 17:3055-3061.

Davidson HJ and Kuonen VJ. 2004. The tear film and ocular mucins. Vet. Ophthalmol. 7:71-77.

Gelatt KN, Gilger BC, Kern TJ. 2013. Veterinary ophthalmology. 5th ed, Wiley-Blackwell, Ames, pp. 171-207.

Gilbard JP, Rossi SR, Gray KL. 1987. A new rabbit model for keratoconjunctivitis sicca. Invest. Ophthalmol. Vis. Sci. 28:225228.

Higuchi A, Ueno R, Shimmura S, Suematsu M, Dogru M, Tsubota K. 2007. Albumin rescues ocular epithelial cells from cell death in dry eye. Curr. Eye Res. 32:83-88.

Jay GD, Britt DE, Cha CJ. 2000. Lubricin is a product of megakaryocyte stimulating factor gene expression by human synovial fibroblasts. J. Rheumatol. 27:594-600.

Jay GD and Hong BS. 1992. Characterization of a bovine synovial fluid lubricating factor. II. Comparison with purified ocular and salivary mucin. Connect. Tissue Res. 28:89-98.

Kim DW, Lee SH, Ku SK, Lee JE, Cha HJ, Youn JK, Kwon HY, Park JH, Park EY, Cho SW, Han KH, Park J, Eum WS, Choi SY. 2015a. The effects of PEP-1-FK506BP on dry eye disease in a rat model. BMB Rep. 48:153-158.

Kim DW, Lee SH, Shin MJ, Kim K, Ku SK, Youn JK, Cho SB, Park JH, Lee CH, Son O, Sohn EJ, Cho SW, Park JH, Kim HA, Han KH, Park J, Eum WS, Choi SY. 2015b. PEP-1-FK506BP inhibits alkali burn-induced corneal inflammation on the rat model of corneal alkali injury. BMB Rep. 48:618-623.

Kuckelkorn R, Schrage N, Redbrake C, Kottek A, Reim M. 1996. Autologous transplantation of nasal mucosa after severe chemical and thermal eye burns. Acta Ophthalmol. Scand. 74:442-448.

Lambiase A, Sullivan BD, Schmidt TA, Sullivan DA, Jay GD, Truitt ER 3rd, Bruscolini A, Sacchetti M, Mantelli F. 2017. A two-week, randomized, double-masked study to evaluate safety and efficacy of lubricin $(150 \mu \mathrm{g} / \mathrm{mL})$ eye drops versus sodium hyaluronate (HA) $0.18 \%$ eye drops $\left(\right.$ Vismed $^{\circledR}$ ) in patients with moderate dry eye disease. Ocul. Surf. 15:77-87.

Laverty S, Girard CA, Williams JM, Hunziker EB, Pritzker KP. 2010. The OARSI histopathology initiative - recommendations for histological assessments of osteoarthritis in the rabbit. Osteoarthritis Cartilage 18 Suppl 3:S53-S65.

Lettesjö H, Nordström E, Ström H, Nilsson B, Glinghammar B, Dahlstedt L, Möller E. 1998. Synovial fluid cytokines in patients with rheumatoid arthritis or other arthritic lesions. Scand. J. Immunol. 48:286-292.

Li N, Deng X, Gao Y, Zhang S, He M, Zhao D. 2013. Establishment of the mild, moderate and severe dry eye models using three methods in rabbits. BMC Ophthalmol. 13:50.

Nakamura S, Shibuya M, Saito Y, Nakashima H, Saito F, Higuchi A, Tsubota K. 2003. Protective effect of D-beta-hydroxybutyrate on corneal epithelia in dry eye conditions through suppression of apoptosis. Invest. Ophthalmol. Vis. Sci. 44:4682-4688.

Plummer CE, Källberg ME, Gelatt KN, Gelatt JP, Barrie KP, Brooks DE. 2008. Intranictitans tacking for replacement of prolapsed gland of the third eyelid in dogs. Vet. Ophthalmol. 11:228-233.

Rhodes M, Heinrich C, Featherstone H, Braus B, Manning S, Cripps PJ, Renwick P. 2012. Parotid duct transposition in dogs: a retrospective review of 92 eyes from 1999 to 2009. Vet. Ophthalmol. 15:213-222.

Saika S, Ikeda K, Yamanaka O, Flanders KC, Nakajima Y, Miyamoto T, Ohnishi Y, Kao WW, Muragaki Y, Ooshima A. 2005. Therapeutic effects of adenoviral gene transfer of bone morphogenic protein-7 on a corneal alkali injury model in mice. Lab. Invest. 85:474-486.

Sanchez RF, Innocent G, Mould J, Billson FM. 2007. Canine keratoconjunctivitis sicca: disease trends in a review of 229 cases. J. Small Anim. Pract. 48:211-217.

Sant' Anna AE, Hazarbassanov RM, de Freitas D, Gomes JÁ. 2012. Minor salivary glands and labial mucous membrane graft in the treatment of severe symblepharon and dry eye in patients with Stevens-Johnson syndrome. Br. J. Ophthalmol. 96:234-239.

Satici A, Bitiren M, Ozardali I, Vural H, Kilic A, Guzey M. 2003. The effects of chronic smoking on the ocular surface and tear characteristics: a clinical, histological and biochemical study. Acta Ophthalmol. Scand. 81:583-587.

Schmidt TA, Sullivan DA, Knop E, Richards SM, Knop N, Liu S, Sahin A, Darabad RR, Morrison S, Kam WR, Sullivan BD. 2013. Transcription, translation, and function of lubricin, a boundary lubricant, at the ocular surface. JAMA Ophthalmol. 131:766-776.

The definition and classification of dry eye disease: report of the Definition and Classification Subcommittee of the International Dry Eye WorkShop (2007). 2007. Ocul. Surf. 5:75-92.

Wang X, Dong J, Wu Q. 2014. Mean central corneal thickness and corneal power measurements in pigmented and white rabbits using Visante optical coherence tomography and ATLAS corneal topography. Vet. Ophthalmol. 17:87-90. 
Ward KW. 2008. Superficial punctate fluorescein staining of the ocular surface. Optom. Vis. Sci. 85:8-16.

Wenkel H, Rummelt V, Naumann GO. 2000. Long term results after autologous nasal mucosal transplantation in severe mucus deficiency syndromes. Br. J. Ophthalmol. 84:279-284.

Wu CL, Chou HC, Li JM, Chen YW, Chen JH, Chen YH, Chan HL. 2013. Hyaluronic acid-dependent protection against alkali-burned human corneal cells. Electrophoresis 34:388396.

Yang G, Espandar L, Mamalis N, Prestwich GD. 2010. A cross- linked hyaluronan gel accelerates healing of corneal epithelial abrasion and alkali burn injuries in rabbits. Vet. Ophthalmol. 13:144-150.

Yokoi N, Bron AJ, Tiffany JM, Maruyama K, Komuro A, Kinoshita S. 2004. Relationship between tear volume and tear meniscus curvature. Arch. Ophthalmol. 122:1265-1269.

Zoukhri D, Fix A, Alroy J, Kublin CL. 2008. Mechanisms of murine lacrimal gland repair after experimentally induced inflammation. Invest. Ophthalmol. Vis. Sci. 49:4399-4406. 